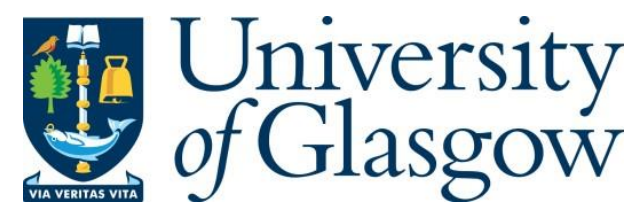

Petermann-Rocha, F., Gray, S. R., Pell, J. P., Ho, F. K. and Celis-Morales, C. (2021) The joint association of sarcopenia and frailty with incidence and mortality health outcomes: a prospective study. Clinical Nutrition, 40(4), pp. 2427-2434.

There may be differences between this version and the published version. You are advised to consult the publisher's version if you wish to cite from it.

$\underline{\text { http://eprints.gla.ac.uk/225834/ }}$

Deposited on: 2 November 2020

Enlighten - Research publications by members of the University of Glasgow http://eprints.gla.ac.uk 


\section{The joint association of sarcopenia and frailty with incidence and mortality health outcomes: a prospective study}

Fanny Petermann-Rocha ${ }^{1,2}$, Stuart R Gray ${ }^{2}$, Jill P Pell ${ }^{1} \dagger$, Frederick K Ho ${ }^{1} \dagger$, Carlos Celis-Morales ${ }^{1,2,3,4}+$

${ }^{1}$ Institute of Health and Wellbeing, University of Glasgow, Glasgow, UK.

${ }^{2}$ British Heart Foundation Glasgow Cardiovascular Research Centre, Institute of Cardiovascular and Medical Sciences, University of Glasgow, Glasgow, UK.

${ }^{3}$ Centre of Exercise Physiology Research (CIFE), Universidad Mayor, Chile.

${ }^{4}$ Laboratorio de Rendimiento Humano, Grupo de Estudio en Educación, Actividad Física y Salud (GEEAFyS), Universidad Católica del Maule, Talca, Chile.

† JPP, FH, CCM contributed equally to this work and are joint senior authors

\section{Corresponding author}

Dr Carlos Celis-Morales

BHF Glasgow Cardiovascular Research Centre

Institute of Cardiovascular and Medical Sciences

College of Medical, Veterinary and Life Sciences

University of Glasgow

Glasgow, G12 8TA

United Kingdom

Tel: + 441413304201

Email: Carlos.Celis@glasgow.ac.uk 


\begin{abstract}
Background: Sarcopenia and frailty are strongly associated with disease incidence and mortality. However, there is limited evidence regarding their joint association with health outcomes. This study aimed to investigate the joint association of sarcopenia and frailty with cardiovascular disease (CVD), respiratory disease and cancer incidence and mortality as well as all-cause mortality in middle-aged and older adults in the UK Biobank study.
\end{abstract}

Methods: 316,980 UK Biobank participants were included in this prospective study (53.1\% women). Sarcopenia was defined according to the EWGSOP2 2019. Frailty was defined using a modified version of the Fried criteria. Combined classifications of sarcopenia and frailty were generated with the following seven subgroups derived: i) normal, ii) non-sarcopenic/pre-frail, iii) non-sarcopenic/frail, iv) pre-sarcopenic/pre-frail, v) pre-sarcopenic/frail, vi) sarcopenic/pre-frail, and vii) sarcopenic/frail. No participants had (pre)sarcopenia but not frailty. Associations between these exposures and health outcomes (incidence and mortality from cardiovascular and respiratory diseases, cancer, as well as, all-cause mortality) were investigated using Cox-proportional hazard models.

Results: $51.7 \%$ of the participants were not sarcopenic nor frail (normal), $41.3 \%$ were pre-frail or frail, $6.5 \%$ pre-sarcopenia and frail (including pre-frail) and $0.5 \%$ as having both sarcopenia and frailty (including pre-frailty). The combination sarcopenic/frail showed the strongest association with CVD (HR: 1.68 [95\% Cl: 1.22 to 2.30]) and respiratory disease incidence (HR: 1.77 [95\% Cl: 1.40 to 2.24]) and for mortality from all-cause (HR: 2.27 [95\% Cl: 1.64 to 3.13]), respiratory disease (HR: 3.50 [95\% $\mathrm{Cl}: 1.97$ to 6.23$]$ ), and cancer (HR: 1.92 [95\% $\mathrm{Cl}: 1.08$ to 3.38]). Finally, when we investigated the associations between the outcomes and exposures by age groups ( $\geq$ and $<60$ years), we identified that, for many outcomes and categories, the associations were higher in younger individuals compared with older adults.

Conclusion: Our findings indicate that different combinations of sarcopenia and frailty were associated with adverse health outcomes, highlighting the joint association between both conditions. However, those individuals with sarcopenia and frailty showed the strongest associations with CVD and respiratory disease incidence and mortality for all-cause and respiratory disease and cancer.

Keywords: Frailty; Sarcopenia; Mortality; Morbidity. 


\section{Background}

Ageing is a complex process characterised by a deterioration in several physiological functions and which results in a gradual loss of function and disability in later life [1]. Of the many deleterious conditions that occur with ageing, particular emphasis has been placed to sarcopenia and frailty as both are multifactorial syndromes associated with falls, hospitalisations, disability, and worse quality of life in older adults. Both conditions can co-exist, but the severity of each may vary, independently of each other, between individuals $[2,3]$.

Frailty is a decline in physiological reserve and multisystem dysregulation along with a functional failure [4]. Clinically, two major operational definitions have been proposed to define frailty: the frailty index and the frailty phenotype (Fried criteria) [4]. The latter has been identified as a straightforward approach to define frailty since it combines five phenotypic criteria (weakness, slowness, physical activity levels, exhaustion, and weight loss) to classify people as normal (or robust), pre-frail, or frail [5]. Sarcopenia, on the other hand, is defined as the age-associated loss of muscle mass and function. Clinically, sarcopenia is identified as low muscle quality and quantity (low grip strength and muscle mass), and when accompanied by low physical performance, the condition is classified as severe [6]. Sarcopenia has been identified as one of the major causes of frailty along with fatigue, polypharmacy and weight loss $[2,3]$.

Sarcopenia and frailty independently predict worse health-related outcomes [7-9]. Studies have shown that frail people and sarcopenic people have a higher risk of morbidity and mortality compared with non-frail or non-sarcopenic people, respectively [7-9]. However, even though previous studies have investigated the relationship and overlap between them $[2,10]$, to our knowledge, only one small study - conducted in 197 older Italian adults between 80 and 85 years - has investigated the joint association between both conditions and all-cause mortality [11]. To date, most of the evidence has been focused on investigating the association of health outcomes with frailty and sarcopenia in older adults, even though it has been recognised that both frailty and sarcopenia can begin earlier in life $[6,9]$. Therefore, this study aimed to investigate the association of combinations of sarcopenia and frailty with cardiovascular diseases (CVD), respiratory disease and cancer outcomes as well as allcause mortality in middle-aged and older adults from UK Biobank.

\section{Methods}

UK Biobank is a very large cohort study based on the general population that recruited over 500,000 participants aged 37-73 years (5.5\% response rate)[12]. In brief, between 2006 and 2010, participants attended one of 22 assessment research centres across Wales, Scotland and England [13, 14]. All participants completed a touch-screen questionnaire, had physical measurements taken, and 
provided blood, urine, and saliva samples at baseline. More information about the UK Biobank protocol can be found online (http://www.ukbiobank.ac.uk)

\section{Sarcopenia definition and measures}

Sarcopenia was defined using the European Working Group on Sarcopenia in Older People 2019 (EWGSOP2) statement [6]. Pre-sarcopenia was defined based on low grip strength, whereas sarcopenia based on low grip strength plus low muscle mass [6]. Severe sarcopenia was defined as having a low strength, low muscle mass and slow gait speed [6]. However, the sarcopenia and severe sarcopenia category were pooled together due to the small number of participants with severe sarcopenia ( $n=182$ ) (hereafter 'sarcopenia').

Grip strength was measured using a Jamar J00105 hydraulic hand dynamometer. One value was taken for each hand, and the average of both values was expressed in absolute units $(\mathrm{kg})$ and used in subsequent analyses. The specific cut-off points by sex are presented in Table 1 [6].

Muscle mass index was derived from skeletal muscle mass $(\mathrm{kg})$ divided by height $(\mathrm{m})$ squared and assessed by trained nurses. To estimate skeletal muscle mass, we used the Janssen equation [15] on the total body composition measured by bioimpedance (BIA, Tanita BC418MA, Tokyo, Japan). The validity of BIA-measured muscle mass was verified using Pearson's correlation coefficient, and a BlandAltman plot (Supplementary Figure 1) against dual-energy X-ray absorptiometry (DXA) measured muscle mass in a subset of UK Biobank participants. The cut-off points by sex are shown in Table 1 [6].

Self-reported walking pace, categorised as slow, average or brisk, was used as a proxy of gait speed. A previous study demonstrated that self-reported walking pace is a good marker of walking speed [16] and strongly associated with adverse health outcomes [17]. To derive a proxy for gait speed, walking pace was dichotomised into slow or normal (average or brisk pace).

In addition, our study was restricted to participants of white-European background due to ethnicrelated differences in body composition and grip strength levels[6, 18].

\section{Frailty definition and measures}

Weight loss, exhaustion, physical activity, walking speed and grip strength are the five criteria used by the frailty phenotype [5]. However, some of these items were adapted to fit the data available within UK Biobank (see Table 1). Therefore, an adapted version of the frailty classification derived by Fried et al. was used in this study.

Weight loss, tiredness/exhaustion, gait speed and grip strength were derived following as described previously by Hanlon et al. [9] (details are presented in supplementary methods and Table 1). Physical 
activity, in turn, was collected using the International Physical Activity Questionnaire (IPAQ) short form [19]. Total physical activity was computed as the sum of walking, moderate and vigorous activity, measured as metabolic equivalents (MET-hours/week). To derive a proxy for the Fried frailty criteria, this variable was categorised into age- and sex-specific quintiles where the lowest quintile (20\%) was classified as fulfilling the physical inactivity criterion for frailty.

Participants were classified as frail if they fulfilled three or more criteria, prefrail if they fulfilled one or two criteria, and robust if they did not fulfil any criteria.

\section{Health outcomes}

The outcomes in the current study were extracted from hospital episodes records for incidence and death register for mortality. The International Classification of Diseases 10 [ICD-10] was used to derive our health outcomes. CVD included the following ICD-10 codes: I20-125, 142.0, 142.6, 142.7, 142.9, 150.0, 150.1, 150.9 and I62-164), respiratory diseases (ICD10: J09-J98), chronic obstructive pulmonary disease [COPD] (ICD10: J40-j44), and all cancer (ICD10: C0-C97, D37- D48) as well as all-cause mortality. Date of death was obtained from death certificates held by the National Health Service (NHS) Information Centre (England and Wales) and the NHS Central Register Scotland (Scotland). Dates and causes of hospital admissions were identified via record linkage to Health Episode Statistics (HES) (England and Wales) and the Scottish Morbidity Records (SMR01) (Scotland). Details of the linkage procedure can be found at http://content.digital.nhs.uk/services. Hospital admissions data were available until June in England and March 2017 in Wales and Scotland. Mortality data were available until June 2020. Therefore, incident disease and mortality follow-up were censored at these dates respectively, or on the date of death, if earlier.

\section{Covariates}

Age at baseline was determined from dates of birth and the date of baseline assessment. Deprivation (area-based socioeconomic status) was resulting from the postcode of residence, using the Townsend score [20]. Never, former or current smoker were the categories used to self-report smoking. The sum of self-reported time spent using a computer, driving, and/or watching television was used to estimate total time spent in sedentary behaviours. Central obesity was calculated from waist circumference, defined as $\geq 88 \mathrm{~cm}$ for women and $\geq 102 \mathrm{~cm}$ for men [21]. Red meat, processed meat intake and hours of sleep were collected through the touch-screen questionnaire at baseline Frequency of alcohol intake was also self-reported at baseline and categorised into 5 categories: daily/almost daily, 3-4 times a week, once/twice a week, 1-3 times a month, special occasions only and never. Medical history (medical doctor diagnosis of depression, stroke, angina, heart attack, hypertension, cancer, diabetes, 
hypertension and COPD) was self-reported. Additional information regading the measurement taken is available on the UK Biobank website (http://www.ukbiobank.ac.uk).

\section{Ethics approval and consent to participate}

UK Biobank was approved by the North West Multi-Centre Research Ethics Committee, and all participants provided written informed consent to participate in the UK Biobank study (Ref: 11/NW/0382). The study protocol is available online (http://www.ukbiobank.ac.uk/).

\section{Statistical analyses}

To determine the joint association between sarcopenia and frailty, the following categories were created: i) normal (no sarcopenia or frailty), ii) non-sarcopenic/pre-frail, iii) non-sarcopenic/frail, iv) pre-sarcopenic/pre-frail, v) pre-sarcopenic/frail, vi) sarcopenic/pre-frail, and vii) sarcopenic/frail. No participants met the criteria for non-frail plus pre- or sarcopenic (Figure 1).

Descriptive characteristics, broken down by the combinations of sarcopenia and frailty, are presented as means with standard deviations (SD) for continuous variables or as percentages for categorical variables. The normality of variables was assessed using the Anderson-Darling test. One-way ANOVA was used to compare the groups. P-values were estimated using the Bonferroni test to avoid inflated type I error.

Associations between the combinations of sarcopenia and frailty with CVD, respiratory disease and cancer disease incidence (fatal and non-fatal) and mortality, as well as all-cause mortality, were investigated using Cox-proportional hazard models. Individuals with neither sarcopenia nor frailty were used as the reference group. The results are reported as hazard ratios (HR) and their 95\% confidence intervals $(95 \% \mathrm{Cls})$. The proportional hazard assumption was checked based on Schoenfeld residuals.

The cumulative crude hazard rate of the mortality outcomes by the sarcopenia and frailty categories and follow-up time were also estimated using the Nelson-Aalen estimator. Finally, to investigate whether the associations between the sarcopenia and frailty categories and the health outcomes differed by age, analyses were stratified by age category ( $<60$ and $\geq 60$ years). An interaction term between age group and the exposure (sarcopenia and frailty categories) was fitted into the Coxregression to test for interaction.

All analyses were performed excluding participants who self-reported medical diagnoses of cancer ( $n=24,197), C V D$ (heart attack or stroke) $(n=9,009)$ and respiratory disease $(n=3,646)$ at baseline when these were included in the outcome. In addition, we run sensitivity analyses using a 2-year landmark 
period where we also excluded participants who experience events with the first two years of followup.

We ran three models for each outcome, including an increasing number of covariates: model 1 was unadjusted. Model 2 was adjusted for socio-demographic covariates (age, sex and deprivation). Model 3 , as per model 2, but additionally adjusted for prevalent diseases (hypertension, diabetes, depression, CVDs, respiratory diseases and cancers when these were not the outcome), lifestyle factors (smoking, sleep duration, total discretionary sedentary time, alcohol, red meat and processed meat intake) and waist circumference at baseline.

Only participants with complete data available for the sarcopenia and frailty categories as well as the covariates were included. STATA 16 statistical software (StataCorp LP) was used to perform the analyses.

\section{Results}

316,980 (63.1\%) white participants with data available for frailty, sarcopenia and covariates were included in this study (Figure 1). The median follow-up period was 10.9 years (interquartile range: 10.6 - 11.6) for disease incidence and 11.2 years (interquartile range: 10.5 - 11.9) for cause-specific and all-cause mortality. Over the follow-up period, 29,136 (9.2\%) participants developed CVD, 48,643 (15.3\%) respiratory diseases, 9,020 (2.8\%) COPD and 37,526 (11.8\%) cancer. In terms of mortality, 16,058 (5.1\%) participants died; 3,019 (1.0\%) from CVD, 2,916 (0.9\%) from respiratory diseases, and $7,460(2.4 \%)$ from cancer.

The cohorts' characteristics by combined categories of sarcopenia and frailty are presented in Table 2. In summary, and compared with people with neither sarcopenia nor frailty (normal), those sarcopenia and with any form of frailty (pre-frail or frail) were older, more deprived and presented a higher prevalence of women. They also had lower physical activity levels, grip strength, and skeletal muscle mass. They also had a higher waist circumference and a higher prevalence of central obesity except for sarcopenic/pre-frail and sarcopenic/frail individuals, those who had a lower waist circumference and lower prevalence of central obesity compared with those apparently normal. The prevalence of CVD, COPD, hypertension, cancer, and depression was higher in these groups compared to people without sarcopenia nor frailty, except for diabetes in the sarcopenic/pre-frail group where the prevalence was lower than those normal.

The associations of sarcopenia and frailty with incident health outcomes are presented in Figure 2 and Supplementary Table 1. For the maximally adjusted model (model 3), all combinations of sarcopenia and frailty were associated with incident CVD. However, those individuals classified as sarcopenic/frail 
presented the highest CVD risk (HR: 1.68 [95\% Cl: 1.22 to 2.30]). Similarly, the magnitude of the association with incidence of respiratory diseases was higher to the extent the categories increased their severity: from the less severe to the more severe combination, i.e., from non-sarcopenic/prefrail to sarcopenic/frail. Except for non-sarcopenic/frail and pre-sarcopenic/frail, all the other combinations were associated with incident COPD, with the combinations of sarcopenic/pre-frail and sarcopenic/frail having the strongest association (HR: 1.76 [95\% Cl: 1.40 to 2.20] and $\mathrm{HR}: 1.63$ [95\% Cl: 1.10 to 2.43$]$ ). No associations were identified between the combinations of sarcopenia and frailty and cancer incidence. Similar trends were overserved when the analyses were performed using a 2year landmark period (Supplementary Table 2).

The joint associations between sarcopenia and frailty and all-cause and cause-specific mortality are shown in Figure 3 and Supplementary Table 3. All categories of sarcopenia and frailty were associated with all-cause mortality; however, those classified as sarcopenic/frail had the highest risk for all-cause mortality (HR: 2.27 [95\% Cl: 1.64 to 3.13]). Similarly, individuals classified in the sarcopenic/frail group had the strongest association with respiratory disease (HR: 3.50 [95\% Cl: 1.97 to 6.23]) and cancer mortality (HR: 1.92 [95\% Cl: 1.08 to 3.38]) (Figure 3). For CVD mortality, those classified as nonsarcopenic/pre-frail, pre-sarcopenic/pre-frail and pre-sarcopenic/pre-frail had 1.16, 1.47 and 1.89fold risk of CVD mortality compared with the reference group (Model 3, Supplementary Table 3). Similar trends were identified when the analyses were performed using a 2-year landmark period (Supplementary Table 4).

When the crude cumulative mortality curves by follow-up time were investigated, we identified that, in comparison to normal individuals, those with any combination of sarcopenia and frailty had a steeper gradient curve (Supplementary Figures 2 to 5). Individuals with both sarcopenia and frailty had the highest mortality rate across age for the four mortality outcomes studied.

Finally, when we investigated whether the associations between the sarcopenia and frailty categories and the adverse outcomes differed by age groups, we identified that, for many outcomes and categories, the associations were higher in younger individuals ( $<60$ years) compared with older adults ( $\geq 60$ years) (Supplementary Tables 5 and 6 ). For instance, we observed that the risk for CVD incidence in younger versus older individuals was 2.9-times and 1.8-times higher, respectively, on those classified as sarcopenic/frail compared to those without these conditions. Larger differences were observed for CVD mortality. Finally, and except for some exceptions, there were no significant interactions between age and the exposure of interest (sarcopenia/frailty categories), (Supplementary Tables 5 and 6). 


\section{Discussion}

Sarcopenia and frailty are associated with each other and often co-exist $[2,3]$. In this study, one of the key findings was evidence of a relationship with all-cause, respiratory disease, and cancer mortality and CVD and respiratory disease incidence across combinations of sarcopenia and frailty from non-sarcopenic/pre-frail to sarcopenic/frail. The association patterns for incident cancer were less clear. The combination of sarcopenic/frail had the strongest association with CVD and respiratory disease incidence as well as all-cause mortality, and respiratory disease and cancer mortality. These results highlight the joint association between both conditions and the poorer prognosis of people who have both conditions. However, we also observed that the risk of adverse health outcomes, especially CVD incidence, associated with sarcopenia and frailty differed by age groups. Compared to individuals without frailty or sarcopenia, those with both conditions who were $\geq 60$ or $<60$ years had a 1.8- and 2.9-times higher risk of CVD incidence, respectively. Although some of the associations were non-significant for some of the combined categories of frailty and sarcopenia in people $<60$ years, the hazard estimates were similar, suggesting that the association between the combination of sarcopenia and frailty among middle-aged adults could be associated with worse health outcomes.

To date, most studies have focused on investigating the independent associations between frailty and all-cause mortality [8], CVD [22], respiratory disease [23], and cancer outcomes [24]; and between sarcopenia and the same health outcomes $[7,25,26]$. However, even though previous studies have demonstrated the higher risk of morbidity and mortality when sarcopenia or frailty are present, to our knowledge, only one studied has highlighted the additive effect of sarcopenia and frailty on the risk of mortality [11]. In that study, Landi et al. demonstrated that frail Italian older adults with sarcopenia had 2.32 times higher risk of death in comparison to people with frailty but without sarcopenia, independently of age and functional variables. However, Landi's study population comprised only 197 frail older adults and sarcopenia was defined using the EWGSOP 2010 (low muscle mass and either low grip strength or slow gait speed) [27]. Therefore, our findings not only support the results of Landi et al. but also test the research question in a larger population and using the new criteria for the diagnosis of sarcopenia of the EWGSOP 2019.

Sarcopenia provides the basis for frailty since two of the frailty components (weakness and slowness) are part of the sarcopenia definition [2]. As a result, it is not surprising that sarcopenia and frailty are overlapping phenotypes and frequently co-exist [28]. The literature has reported different measurement and classifications to diagnose them $[5,6,28]$; however, in spite of the strong associations identified, they are rarely measured in clinical practice. In fact, only $41.6 \%$ of health professionals who were members of the Association of Rehabilitation Nutrition in Japan reported 
measuring the physical capability markers necessary to diagnose sarcopenia [29]. Nevertheless, if we take into account that both syndromes are potentially reversible and are not inevitable during ageing $[2,3]$ both the risk of morbidity and mortality as well as the burden of disability and hospitalisations in older adults could be reduced.

\section{Strengths and limitations}

UK Biobank is not representative of the UK population in terms of socio-demographic, lifestyle and prevalent disease [30]. Therefore, whilst estimates of effect sizes can be generalised, summary statistics should not be [31]. The use of UK Biobank provided the opportunity to test our research question in a very large general population cohort as well as the opportunity to work with information collected using validated and standardised methods. Moreover, the frailty phenotype was created using similar, but not the original variables suggested by Fried et al. [5]. In addition, four out five of the variables used to define frailty were self-reported. Although this approach has shown to be comparable with quantitative or objective measures [32], some variables could be affected by reporting bias. In terms of sarcopenia, muscle mass was measured using bioimpedance. Even if this method is not the gold standard in terms of measuring muscle, muscle mass estimated using BIA demonstrated to have a good level of agreement with DXA ( $r=0.868, p<0.0001$; Supplementary Figure 1). In addition, despite sarcopenic/frail had a significant association with several health outcomes included in this study, the lack of association for some outcomes may be due to lack of power, as a lower number of event or cases were available. These results are not unexpected if we consider that both sarcopenia and frailty are ageing-related diseases with an onset later in life and that UK Biobank population is a relatively young with a small proportion of older or very old adults. Therefore, the analyses should be repeated in an even larger individual study, or else in another study and combined in a meta-analysis. Finally, although we adjust our models for a comprehensive list of confounding factors, we also need to consider that some of the associations observed may be due to residual confounding or unmeasured confounding factors.

\section{Conclusion}

Our findings demonstrated that the combination of sarcopenic/frail was associated with adverse health outcomes, with the strongest risk observed for CVD and respiratory disease incidence as well as all-cause mortality, and respiratory disease and cancer mortality. Considering that both syndromes could be assessed in clinical practice, consideration should be given to screening both middle-aged and older individuals of the general population to identify and treat these conditions early in their natural history. 


\section{Funding}

UK Biobank was established by the Wellcome Trust medical charity, Medical Research Council, Department of Health, Scottish Government and the Northwest Regional Development Agency. It has also had funding from the Welsh Assembly Government and the British Heart Foundation. All authors had final responsibility for submission for publication. FP-R receives financial support from the Chilean Government for doing her PhD (ANID-Becas Chile 2018 - 72190067).

\section{Conflict of interest}

The authors declare no conflict of interest.

\section{Acknowledgements}

This research has been conducted using the UK Biobank resource. We are grateful to UK Biobank participants. This research has been conducted using the UK Biobank resource under application number 7155. 


\section{References}

[1] Lara J, Sherratt MJ, Rees M. Aging and anti-aging. Maturitas. 2016;93:1-3.

[2] Cesari M, Landi F, Vellas B, Bernabei R, Marzetti E. Sarcopenia and physical frailty: two sides of the same coin. Front Aging Neurosci. 2014;6:192-.

[3] Dodds R, Sayer AA. Sarcopenia and frailty: new challenges for clinical practice. Clin Med (Lond). 2016;16:455-8.

[4] WHO. WHO Clinical Consortium on Healthy Ageing. Topic focus: frailty and intrinsic capacity. World Health Organization. 2016;Available at:

https://apps.who.int/iris/bitstream/handle/10665/272437/WHO-FWC-ALC-17.2eng.pdf? sequence $=1$ \&isAllowed $=\mathrm{y}$.

[5] Fried LP, Tangen CM, Walston J, Newman AB, Hirsch C, Gottdiener J, et al. Frailty in older adults: evidence for a phenotype. J Gerontol A Biol Sci Med Sci. 2001;56:M146-M56.

[6] Cruz-Jentoft AJ, Bahat G, Bauer J, Boirie Y, Bruyere O, Cederholm T, et al. Sarcopenia: revised European consensus on definition and diagnosis. Age and ageing. 2019;48:16-31.

[7] Liu P, Hao Q, Hai S, Wang H, Cao L, Dong B. Sarcopenia as a predictor of all-cause mortality among community-dwelling older people: A systematic review and meta-analysis. Maturitas. 2017;103:16-22.

[8] Li X, Ploner A, Karlsson IK, Liu X, Magnusson PKE, Pedersen NL, et al. The frailty index is a predictor of cause-specific mortality independent of familial effects from midlife onwards: a large cohort study. BMC Medicine. 2019;17:94.

[9] Hanlon P, Nicholl BI, Jani BD, Lee D, McQueenie R, Mair FS. Frailty and pre-frailty in middle-aged and older adults and its association with multimorbidity and mortality: a prospective analysis of 493737 UK Biobank participants. Lancet Public Health. 2018;3:e323-e32.

[10] Bone AE, Hepgul N, Kon S, Maddocks M. Sarcopenia and frailty in chronic respiratory disease. Chron Respir Dis. 2017;14:85-99.

[11] Landi F, Cruz-Jentoft AJ, Liperoti R, Russo A, Giovannini S, Tosato M, et al. Sarcopenia and mortality risk in frail older persons aged 80 years and older: results from ilSIRENTE study. Age and Ageing. 2013;42:203-9.

[12] Collins R. What makes UK Biobank special? Lancet (London, England). 2012;379:1173-4.

[13] Palmer L. UK Biobank: bank on it. Lancet (London, England). 2007;369:1980-2.

[14] Sudlow C, Gallacher J, Allen N, Beral V, Burton P, Danesh J, et al. UK biobank: an open access resource for identifying the causes of a wide range of complex diseases of middle and old age. PLoS medicine. 2015;12:e1001779.

[15] Janssen I, Heymsfield SB, Baumgartner RN, Ross R. Estimation of skeletal muscle mass by bioelectrical impedance analysis. Journal of applied physiology (Bethesda, Md : 1985). 2000;89:46571.

[16] Syddall HE, Westbury LD, Cooper C, Sayer AA. Self-reported walking speed: a useful marker of physical performance among community-dwelling older people? J Am Med Dir Assoc. 2015;16:3238.

[17] Ganna A, Ingelsson E. 5 year mortality predictors in 498103 UK Biobank participants: a prospective population-based study. The Lancet. 2015;386:533-40.

[18] Chen L-K, Woo J, Assantachai P, Auyeung T-W, Chou M-Y, lijima K, et al. Asian Working Group for Sarcopenia: 2019 Consensus Update on Sarcopenia Diagnosis and Treatment. Journal of the American Medical Directors Association.

[19] Guo W, Bradbury KE, Reeves GK, Key TJ. Physical activity in relation to body size and composition in women in UK Biobank. Annals of epidemiology. 2015;25:406-13.e6.

[20] Townsend P PM, Beattie A. Health and deprivation. Inequality and the North. Health Policy (New York). 1988;10.

[21] WHO. Obesity: preventing and managing the global epidemic. Report of a WHO consultation. World Health Organization technical report series2000. p. i-xii, 1-253. 
[22] Veronese N, Sigeirsdottir K, Eiriksdottir G, Marques EA, Chalhoub D, Phillips CL, et al. Frailty and Risk of Cardiovascular Diseases in Older Persons: The Age, Gene/Environment SusceptibilityReykjavik Study. Rejuvenation Res. 2017;20:517-24.

[23] Lahousse L, Ziere G, Verlinden VJA, Zillikens MC, Uitterlinden AG, Rivadeneira F, et al. Risk of Frailty in Elderly With COPD: A Population-Based Study. J Gerontol A Biol Sci Med Sci. 2016;71:68995.

[24] Brown JC, Harhay MO, Harhay MN. The Prognostic Importance of Frailty in Cancer Survivors. J Am Geriatr Soc. 2015;63:2538-43.

[25] Locquet M, Beaudart C, Petermans J, Reginster J-Y, Bruyère O. EWGSOP2 Versus EWGSOP1: Impact on the Prevalence of Sarcopenia and Its Major Health Consequences. Journal of the American Medical Directors Association. 2019;20:384-5.

[26] Petermann-Rocha F, Chen M, Gray SR, Ho FK, Pell JP, Celis-Morales C. New versus old guidelines for sarcopenia classification: What is the impact on prevalence and health outcomes? Age and ageing. 2019:afz126.

[27] Cruz-Jentoft AJ, Baeyens JP, Bauer JM, Boirie Y, Cederholm T, Landi F, et al. Sarcopenia: European consensus on definition and diagnosis: Report of the European Working Group on Sarcopenia in Older People. Age and ageing. 2010;39:412-23.

[28] Keevil VL, Romero-Ortuno R. Ageing well: a review of sarcopenia and frailty. Proceedings of the Nutrition Society. 2015;74:337-47.

[29] Nakahara S, Wakabayashi H, Maeda K, Nishioka S, Kokura Y. Sarcopenia and cachexia evaluation in different healthcare settings: a questionnaire survey of health professionals. Asia Pac J Clin Nutr. 2018;27:167-75.

[30] Fry A, Littlejohns TJ, Sudlow C, Doherty N, Adamska L, Sprosen T, et al. Comparison of Sociodemographic and Health-Related Characteristics of UK Biobank Participants With Those of the General Population. American journal of epidemiology. 2017;186:1026-34.

[31] Batty GD, Gale CR, Kivimäki M, Deary IJ, Bell S. Comparison of risk factor associations in UK Biobank against representative, general population based studies with conventional response rates: prospective cohort study and individual participant meta-analysis. BMJ. 2020;368:m131.

[32] Theou O, O'Connell MDL, King-Kallimanis BL, O'Halloran AM, Rockwood K, Kenny RA. Measuring frailty using self-report and test-based health measures. Age and ageing. 2015;44:471-7. 
Table 1. Frailty and sarcopenia definition and cut-off points

\begin{tabular}{|c|c|c|}
\hline $\begin{array}{l}\text { Individuals } \\
\text { components }\end{array}$ & $\begin{array}{c}\text { Frailty (adapted from Halton et al. [9] and } \\
\text { used in this manuscript) }\end{array}$ & $\begin{array}{c}\text { Sarcopenia (Adapted from the } \\
\text { EWGSOP2 [6] and used in this } \\
\text { manuscript ) }\end{array}$ \\
\hline Weight Loss & $\begin{array}{l}\text { Self-reported: "Compared with one year } \\
\text { ago, has your weight changed?" } \\
\text { Options: } \\
\text { Yes: weight loss in the previous year. } \\
\text { No: another option. }\end{array}$ & $\mathrm{N} / \mathrm{A}$ \\
\hline Exhaustion & $\begin{array}{l}\text { Self-reported: "Over the past two weeks, } \\
\text { how often have you felt tired or had little } \\
\text { energy" } \\
\text { Options: } \\
\text { Yes: more than half time or every day } \\
\text { No: another option }\end{array}$ & $\mathrm{N} / \mathrm{A}$ \\
\hline $\begin{array}{l}\text { Low physical } \\
\text { activity }\end{array}$ & $\begin{array}{l}\text { Quintiles of sex- age-specific levels of total } \\
\text { PA in derived from IPAQ } \\
\text { Options: } \\
\text { Yes: Lowest level of PA } \\
\text { No: low/middle to highest levels of PA }\end{array}$ & $\mathrm{N} / \mathrm{A}$ \\
\hline Slow walking speed & $\begin{array}{l}\text { Self-reported: How do you describe your } \\
\text { usual walking pace? (a proxy for gait speed) } \\
\text { Options: } \\
\text { Yes: slow } \\
\text { No: average or brisk pace }\end{array}$ & $\begin{array}{l}\text { Self-reported: How do you describe } \\
\text { your usual walking pace? (a proxy for } \\
\text { gait speed) } \\
\text { Options: } \\
\text { Yes: slow } \\
\text { No: average or brisk pace }\end{array}$ \\
\hline Low grip strength & $\begin{array}{l}\text { Measured grip strength expressed in } \mathrm{kg} \text { by } \\
\text { sex-and BMI adjusted cut-off points. } \\
\text { Cut-off points: } \\
\text { The same proposed for Fried et al.[5]. }\end{array}$ & $\begin{array}{l}\text { Average of the right and left hand } \\
\text { expressed in absolute units (kg). } \\
\text { Cut-off points: } \\
\text { Men: }<27 \mathrm{~kg} \\
\text { Women: }<16 \mathrm{~kg}\end{array}$ \\
\hline Low muscle mass & N/A & $\begin{array}{l}\text { Derived from appendicular lean } \\
\text { muscle mass }(\mathrm{kg}) \text { divided by height }(\mathrm{m}) \\
\text { squared using the Janssen } \\
\text { equation[15]. } \\
\text { Cut-off points: } \\
\text { Men: }<7.0 \mathrm{~kg} / \mathrm{m}^{2} \\
\text { Women: }<5.5 \mathrm{~kg} / \mathrm{m}^{2}\end{array}$ \\
\hline
\end{tabular}

Table adapted from Halton et al. and the EWGSOP2 [6, 9]. N/A: non-applicable. 
Table 2. Baseline characteristic by frailty and sarcopenia status

\begin{tabular}{|c|c|c|c|c|c|c|c|}
\hline & Normala $^{a}$ & $\begin{array}{c}\text { Non-sarcopenic/ } \\
\text { pre-frail }^{b}\end{array}$ & $\begin{array}{c}\text { Non-sarcopenic/ } \\
\text { frailc }\end{array}$ & $\begin{array}{c}\text { Pre-sarcopenic/ } \\
\text { pre-frail d }^{\text {d }}\end{array}$ & $\begin{array}{c}\text { Pre-sarcopenic/ } \\
\text { fraile }^{\text {en }}\end{array}$ & $\begin{array}{l}\text { Sarcopenic/ } \\
\text { pre-frail*f }\end{array}$ & $\begin{array}{l}\text { Sarcopenic/ } \\
\text { frail }^{* g}\end{array}$ \\
\hline \multicolumn{8}{|l|}{ Socio-demographics } \\
\hline Total n, (\%) & $163,797(51.7)$ & $127,812(40.3)$ & $3,165(1.0)$ & $19,177(6.0)$ & $1,656(0.5)$ & $1,161(0.4)$ & $212(0.1)$ \\
\hline Age at baseline (years), mean (SD) & $56.2(8.1)^{\mathrm{d}, \mathrm{e}, \mathrm{f}, \mathrm{g}}$ & $56.2(8.1)^{d, e, f, g}$ & $56.3(8.0)^{\mathrm{d}, \mathrm{e}, \mathrm{f}, \mathrm{g}}$ & $60.4(7.0)^{a, b, c, e, f}$ & $58.6(7.4)^{a, b, c, c, d, f, g}$ & $63.2(5.0)^{a, b, c, d, e}$ & $61.8(6.3)^{\mathrm{a}, \mathrm{b}, \mathrm{c}, \mathrm{e}}$ \\
\hline Sex (women), n (\%) & $\begin{array}{c}82,113(50.1) \\
b, c, d, e, f, g\end{array}$ & $\begin{array}{c}69,912(54.7) \\
a, c, d, e, f, g\end{array}$ & $\begin{array}{c}2,060(65.1) \\
a, b, f, g\end{array}$ & $\begin{array}{c}12,160(63.4) \\
a, b, e, f, g\end{array}$ & $\begin{array}{c}1,143(69.0) \\
a, b, d, d, f, f\end{array}$ & $\begin{array}{c}1,109(95.5) \\
a, b, c, c, d, e\end{array}$ & $\begin{array}{c}199(93.4) \\
a, b, c, d, e\end{array}$ \\
\hline Deprivation, n (\%) & $b, c, d, e, f, g$ & $\mathrm{a}, \mathrm{c}, \mathrm{d}, \mathrm{e}$ & $a, f$ & $\mathrm{a}, \mathrm{b}, \mathrm{e}$ & $\mathrm{a}, \mathrm{b}, \mathrm{d}, \mathrm{f}$ & $\mathrm{a}, \mathrm{c}, \mathrm{e}$ & $\mathrm{a}$ \\
\hline Lower & $61,097(37.3)$ & $44,684(35.0)$ & $940(29.7)$ & $6,048(31.5)$ & $447(27.0)$ & $375(32.3)$ & $60(28.3)$ \\
\hline Middle & $57,539(35.1)$ & $43,912(34.4)$ & $1,046(33.0)$ & $6,491(33.9)$ & $524(31.7)$ & $433(37.3)$ & $77(36.3)$ \\
\hline Higher & $45,161(27.6)$ & $39,216(30.6)$ & $1,179(37.3)$ & $6,638(34.6)$ & $685(41.3)$ & $353(30.4)$ & $75(35.4)$ \\
\hline \multicolumn{8}{|l|}{ Obesity-related markers } \\
\hline Waist Circumference (cm) & $\begin{array}{c}88.0(12.4) \\
b, c, d, e, f, f, g\end{array}$ & $90.7(13.0)^{a, c, d, e, f, g}$ & $\begin{array}{c}94.6(13.5) \\
a, b, d, e, f\end{array}$ & $\begin{array}{c}89.3(12.8) \\
a, b, c, c, e, g\end{array}$ & $\begin{array}{c}90.4(13.5) \\
a, c, d, d, f, g\end{array}$ & $\begin{array}{c}78.2(8.8) \\
a, b, c, e, g\end{array}$ & $\begin{array}{c}79.9(10.0) \\
\text { a,b,d,e,f }\end{array}$ \\
\hline Central Obesity, n (\%) & $\begin{array}{c}39,074(23.9) \\
b, c, d, e, f\end{array}$ & $\begin{array}{c}45,769 \\
(35.8), c, e, f, g\end{array}$ & $\begin{array}{c}1,719(54.3) \\
\text { a,b,d,e,f,g }\end{array}$ & $\begin{array}{c}6,824(35.6) \\
\text { a,ce,f,fg }\end{array}$ & $\begin{array}{c}709(42.8) \\
a, b, c, d, e, g\end{array}$ & $\begin{array}{c}152(13.1) \\
a, b, c, d, e\end{array}$ & $\begin{array}{c}43(20.3) \\
b, c, d, e\end{array}$ \\
\hline Skeletal muscle mass (kg), mean (SD) & $23.0(6.0)^{b, c, d, e, f, g}$ & $22.6(6.0)^{b, c, d, e, f, g}$ & $21.6(5.5)^{a, b, d, e, f, g}$ & $20.4(5.4)^{a, b, c, e, f, g}$ & $20.1(5.3)^{a, b, c, d, f, g}$ & $13.9(1.9)^{a, b, c, d, e}$ & $14.1(2.0)^{a, b, c, d, e}$ \\
\hline \multicolumn{8}{|l|}{ Lifestyle } \\
\hline Total PA (MET/h/week), mean (SD) & $\begin{array}{c}3,531.5(3,128.6) \\
\text { b,c,d,e,f,g }\end{array}$ & $\begin{array}{c}2,217.9 \\
(2,892.0)^{a, c, d, e, f, g}\end{array}$ & $\begin{array}{c}882(1,673.5) \\
a, b, d, e, f\end{array}$ & $\begin{array}{c}2,87.1(2,979.4) \\
\text { a,b,c,e,e,g }\end{array}$ & $\begin{array}{c}1,160.7(2,075.4) \\
a, b, c, d, f f\end{array}$ & $\begin{array}{c}2,762.2 \\
(2,637.1)^{\mathrm{a}, \mathrm{b}, \mathrm{c}, \mathrm{d}, \mathrm{g}}\end{array}$ & $\begin{array}{c}806.9(938.2) \\
\text { a,b,d,f }\end{array}$ \\
\hline Grip Strength (kg) mean (SD) & $34.0(10.1)^{b, c, d, e, f, g}$ & $31.0(10.2)^{a, c, d, d, f, g}$ & $24.3(7.9)^{a, b, d, e, f, g}$ & $16.3(5.7)^{\mathrm{a}, \mathrm{b}, \mathrm{c}, \mathrm{e}, \mathrm{f}, \mathrm{g}}$ & $15.4(5.6)^{a, b, c, d, f, g}$ & $12.7(3.7)^{a, b, c, d, e}$ & $11.5(4.7)^{\mathrm{a}, \mathrm{b}, \mathrm{c}, \mathrm{d}, \mathrm{e}}$ \\
\hline Total Sedentary behaviour (h/day), mean (SD) & $4.8(2.0)^{b, c, d, e, f}$ & $5.0(2.2)^{a, c, d, f}$ & $5.3(2.4)^{a, b, d, e, f}$ & $4.9(2.1)^{a, b, c, e, f}$ & $5.1(2.4)^{a, c, d, f}$ & $4.6(1.9)^{a, b, c, d, e}$ & $5.1(2.4)$ \\
\hline Red meat (portion/week, mean (SD) & $2.1(1.4)$ & $2.1(1.4)$ & $2.0(1.4)$ & $2.1(1.4)$ & $2.0(1.5)$ & $2.0(1.3)$ & $2.0(1.6)$ \\
\hline $\begin{array}{l}\text { Processed meat intake (portion/week), mean } \\
\text { (SD) }\end{array}$ & $1.9(1.0)^{b, f, g}$ & $1.9(1.0)^{\mathrm{a}, \mathrm{d}, \mathrm{f}, \mathrm{g}}$ & $1.9(1.1)^{\mathrm{d}, \mathrm{e}, \mathrm{f}, \mathrm{g}}$ & $1.8(1.0)^{\mathrm{b}, \mathrm{c}, \mathrm{f}, \mathrm{g}}$ & $1.8(1.1)^{c, f}$ & $1.6(1.0)^{\mathrm{a}, \mathrm{b}, \mathrm{c}, \mathrm{d}, \mathrm{e}}$ & $1.6(1.0)^{\mathrm{a}, \mathrm{b}, \mathrm{c}, \mathrm{d}}$ \\
\hline
\end{tabular}




\begin{tabular}{|c|c|c|c|c|c|c|c|}
\hline Alcohol frequency intake, n (\%) & $\mathrm{b}, \mathrm{c}, \mathrm{d}, \mathrm{d}, \mathrm{e}, \mathrm{f}, \mathrm{g}$ & $\mathrm{a}, \mathrm{c}, \mathrm{d}, \mathrm{e}, \mathrm{f}, \mathrm{g}$ & $a, b, d, e, g$ & $a, b, c, e, f, g$ & $a, b, c, d, f$ & $a, b, d, e, g$ & $a, b, c, d, f$ \\
\hline Daily or almost daily & $39,557(24.2)$ & $26,378(20.6)$ & $482(15.2)$ & 3,705 (19.3) & $235(14.1)$ & $230(19.8)$ & $32(15.1)$ \\
\hline 3-4 times a week & $44,523(27.2)$ & $30,676(24.0)$ & $551(17.4)$ & $4,053(21.1)$ & $259(15.8)$ & $198(17.1)$ & $24(11.3)$ \\
\hline Once or twice a week & $42,637(26.0)$ & $34,481(27.0)$ & $828(26.2)$ & $5,027(26.2)$ & $430(26.0)$ & $271(23.3)$ & $51(24.1)$ \\
\hline 1-3 times a month & $16,209(9.9)$ & $14,886(11.7)$ & 474 (14.9) & $2,083(10.9)$ & $229(13.8)$ & $152(13.1)$ & $21(9.9)$ \\
\hline Special occasions only & $13,119(8.0)$ & $13,577(10.6)$ & $519(16.4)$ & $2,554(13.3)$ & 297 (17.9) & $185(16.0)$ & $45(21.2)$ \\
\hline Never & $7,752(4.7)$ & $7,814(6.1)$ & $311(9.9)$ & $1,755(9.2)$ & $206(12.4)$ & $125(10.7)$ & $39(18.4)$ \\
\hline Smoking status n (\%) & $\mathrm{b}, \mathrm{c}, \mathrm{e}$ & $\mathrm{a}, \mathrm{d}, \mathrm{f}$ & $a, d, f$ & $b, c, e$ & $\mathrm{a}, \mathrm{d}, \mathrm{f}$ & $\mathrm{b}, \mathrm{c}, \mathrm{e}, \mathrm{g}$ & $f$ \\
\hline Never & $90,867(55.5)$ & $69,359(54.3)$ & $1,702(53.7)$ & $10,650(55.6)$ & $878(53.0)$ & $693(59.8)$ & $106(50.0)$ \\
\hline Previous & $58,375(35.6)$ & 45,897 (35.9) & 1,079 (34.1) & $6,947(36.2)$ & $562(33.9)$ & $363(31.2)$ & $75(35.4)$ \\
\hline Current & $14,555(8.9)$ & $12,566(9.8)$ & $384(12.2)$ & $1,580(8.2)$ & $216(13.1)$ & $105(9.0)$ & $31(14.6)$ \\
\hline Sleep time (h/day), mean (SD) & $7.2(1.0)^{b, c, d, e}$ & $7.1(1.1)^{\mathrm{a}, \mathrm{c}}$ & $7.0(1.4)^{a, b, d, f}$ & $7.1(1.2)^{a, c, e}$ & $7.0(1.5)^{\mathrm{a}, \mathrm{d}, \mathrm{f}}$ & $7.2(1.2)^{\mathrm{c}, \mathrm{e}}$ & $7.2(1.7)$ \\
\hline \multicolumn{8}{|l|}{ Health status, $\mathrm{n}(\%)$} \\
\hline Diabetes history & $4,082(2.5)^{b, c, d, d e}$ & $6,385(5.0)^{a, c, d, d, e, f}$ & $349(11.0)^{a, b, d, f, g}$ & $1,241(6.5)^{a, b, c, e, e, f}$ & $187(11.2)^{a, b, d, f, g}$ & $20(1.7)^{b, c, d, d, e}$ & $7(3.3)^{\mathrm{ce} e}$ \\
\hline CVDs history & $38,880(23.7)^{b, c, d, e, g}$ & $\begin{array}{c}38,239(29.9) \\
a, c, d, e\end{array}$ & $1,260(39.8)^{a, b, d, f f}$ & $\begin{array}{c}6,557(34.2) \\
a, b, c, c, e, f\end{array}$ & $652(39.4)^{a, b, d, f}$ & $309(26.6)^{c, d, e, g}$ & $78(36.8)^{a, f}$ \\
\hline High blood pressure history & $32,538(19.9)^{b, c, d, e}$ & $31,798(24.9)^{a, c, d, e}$ & $1,012(32.0)^{a, b, e, f}$ & $5,144(26.8)^{a, b, f}$ & $497(30.0)^{a, b, c, f}$ & $246(21.2)^{c, d, e}$ & $49(23.1)$ \\
\hline COPD history & $1,478(0.9)^{b, c, e}$ & $1,608(1.3)^{a, d, f}$ & $81(2.6)^{a, d, f}$ & $353(1.8)^{b, c, e}$ & $62(3.7)^{a, d, f}$ & $49(4.2)^{b, c, e, g}$ & $15(7.1)^{f}$ \\
\hline Cancer history & $11,665(7.1)^{b, c, d, e, f, g}$ & $9,959(7.8)^{a, d, e, f, g}$ & $291(9.2)^{a, f, g}$ & $1,941(10.1)^{a, b, f, g}$ & $167(10.1)^{a, b, g}$ & $148(12.7)^{a, b, c, d}$ & $36(17.0)^{a, b, c, d, e}$ \\
\hline Depression history & $6,029(3.7)^{b, c, d, e, g}$ & $8,196(6.4)^{\mathrm{a}, \mathrm{c}, \mathrm{e}, \mathrm{g}}$ & $433(13.6)^{a, b, d, f}$ & $1,134(5.9)^{a, c, e, g}$ & $200(12.1)^{a, b, d, f}$ & $60(5.2)^{c, e, g}$ & $24(11.3)^{a, b, d, f}$ \\
\hline
\end{tabular}

BMI: body mass index; $\mathrm{n}$ : number; PA: physical activity; MET: metabolic-equivalent; SD: standard deviation. People without sarcopenia or frailty were labelled as normal in the table. *Sarcopenic people include those with sarcopenia or severe sarcopenia.

One-way ANOVA was used to compare the groups. P-values were estimated using the Bonferroni method. The letters a, b, c, d, e, f, and g indicate those variables that were significantly different between groups ( $p$-value $<0.05$ ). For categorical variables, the effect was estimated for the whole group. 


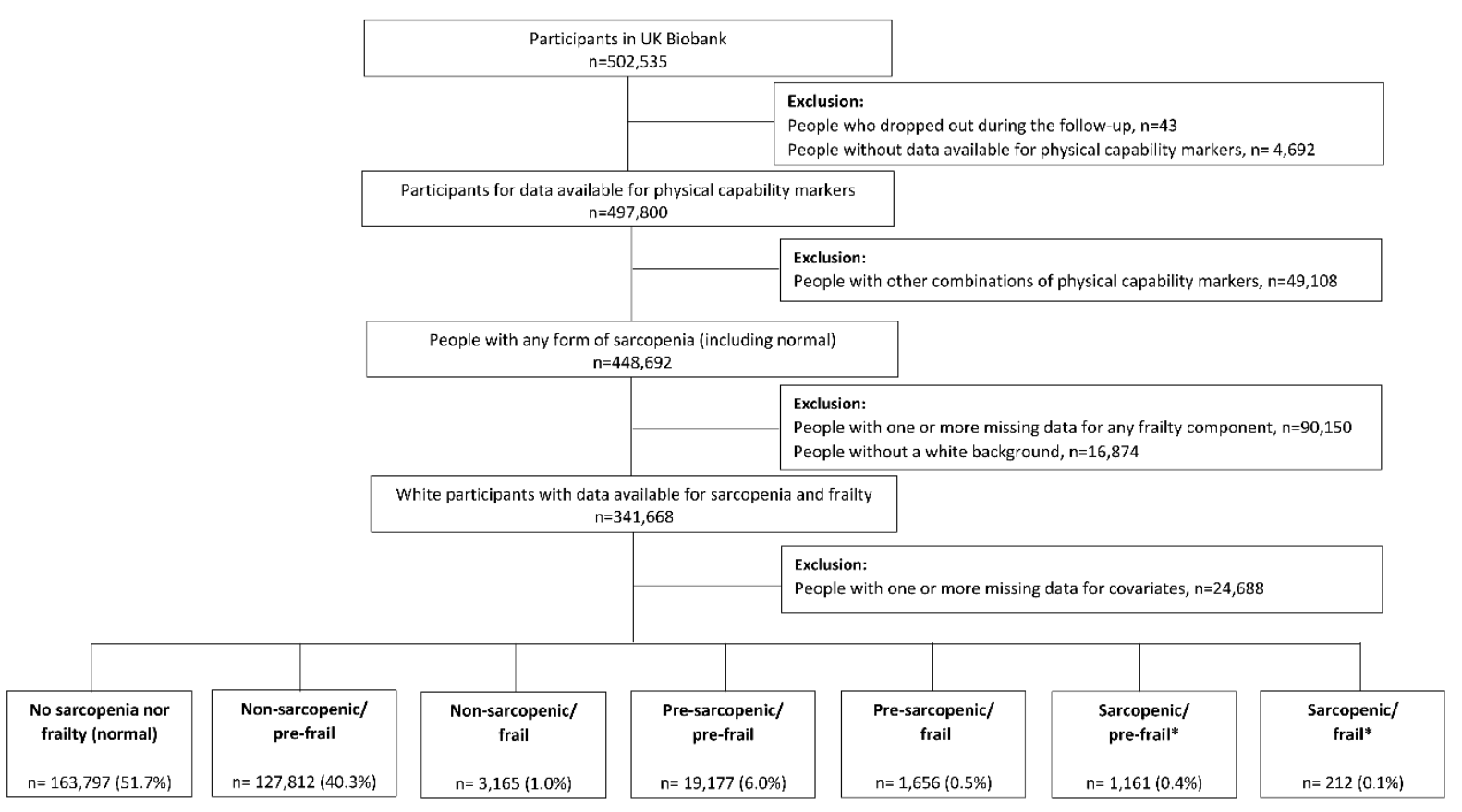

Figure 1. Diagram - Participants according to the different combinations of sarcopenia and frailty in the Uk Biobank.

* Sarcopenic people include those with sarcopenia or severe sarcopenia. 


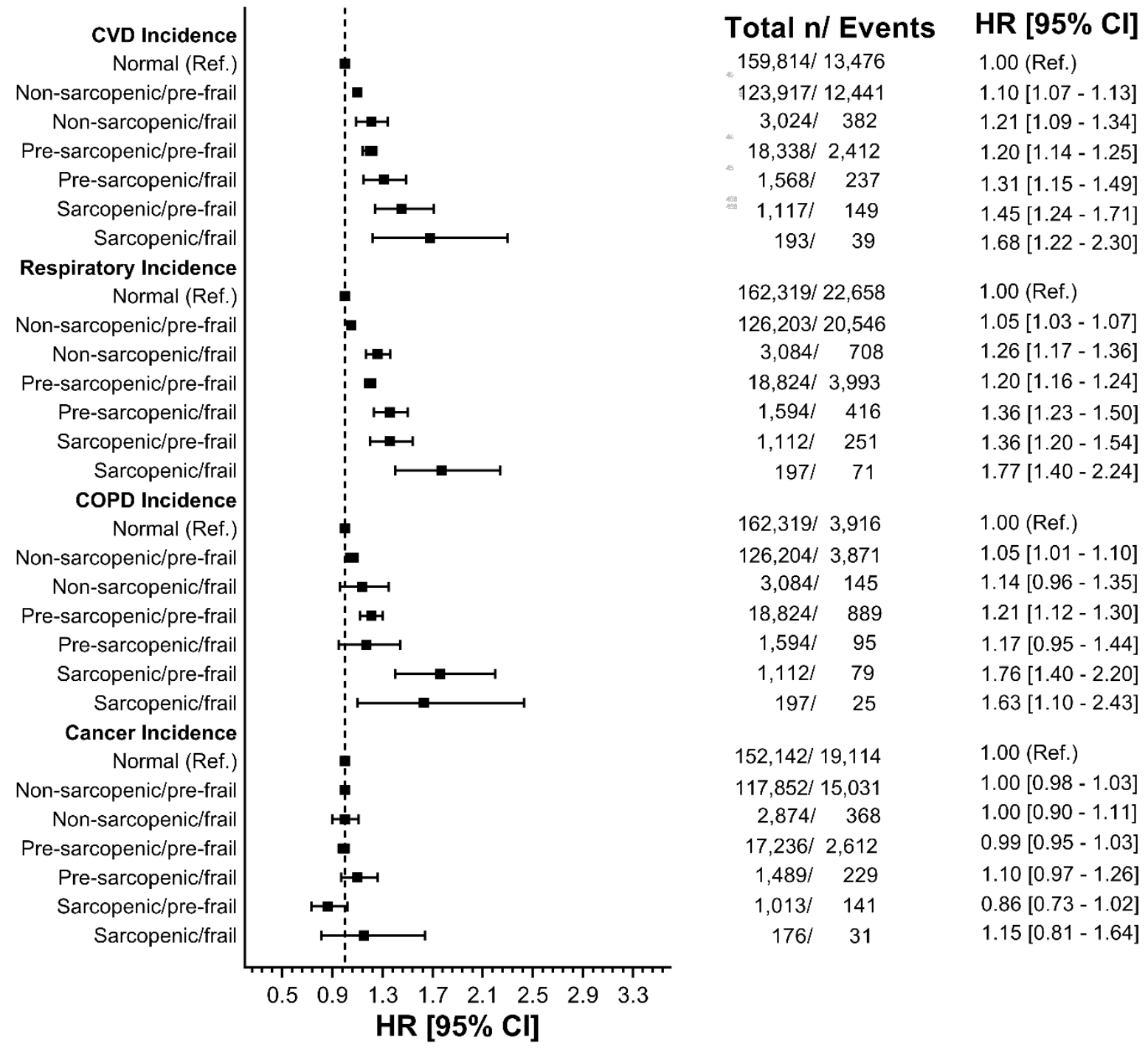

Figure 2. The joint association of frailty and sarcopenia with cause-specific incidence

Data presented as adjusted hazard ratio (HR) and its $95 \%$ confidence interval $(95 \% \mathrm{Cl})$ by combined categories of sarcopenia and frailty. People without sarcopenia or frailty were used as the reference group (label as normal in the graph).

All analyses were conducted excluding people with cancer $(n=24,197), \operatorname{CVD}(n=9,009)$ and respiratory disease $(n=3,646)$ at baseline when these were included in the outcome. Analyses were adjusted for age, sex, deprivation, smoking status, sleep duration, discretionary sedentary time, waist circumference, dietary intake (alcohol, red meat and processed meat intake), systolic blood pressure, diabetes, depression, comorbidities, as well as CVD, cancer, and respiratory disease, when these were not the outcome. Sarcopenic people include those with sarcopenia or severe sarcopenia. 


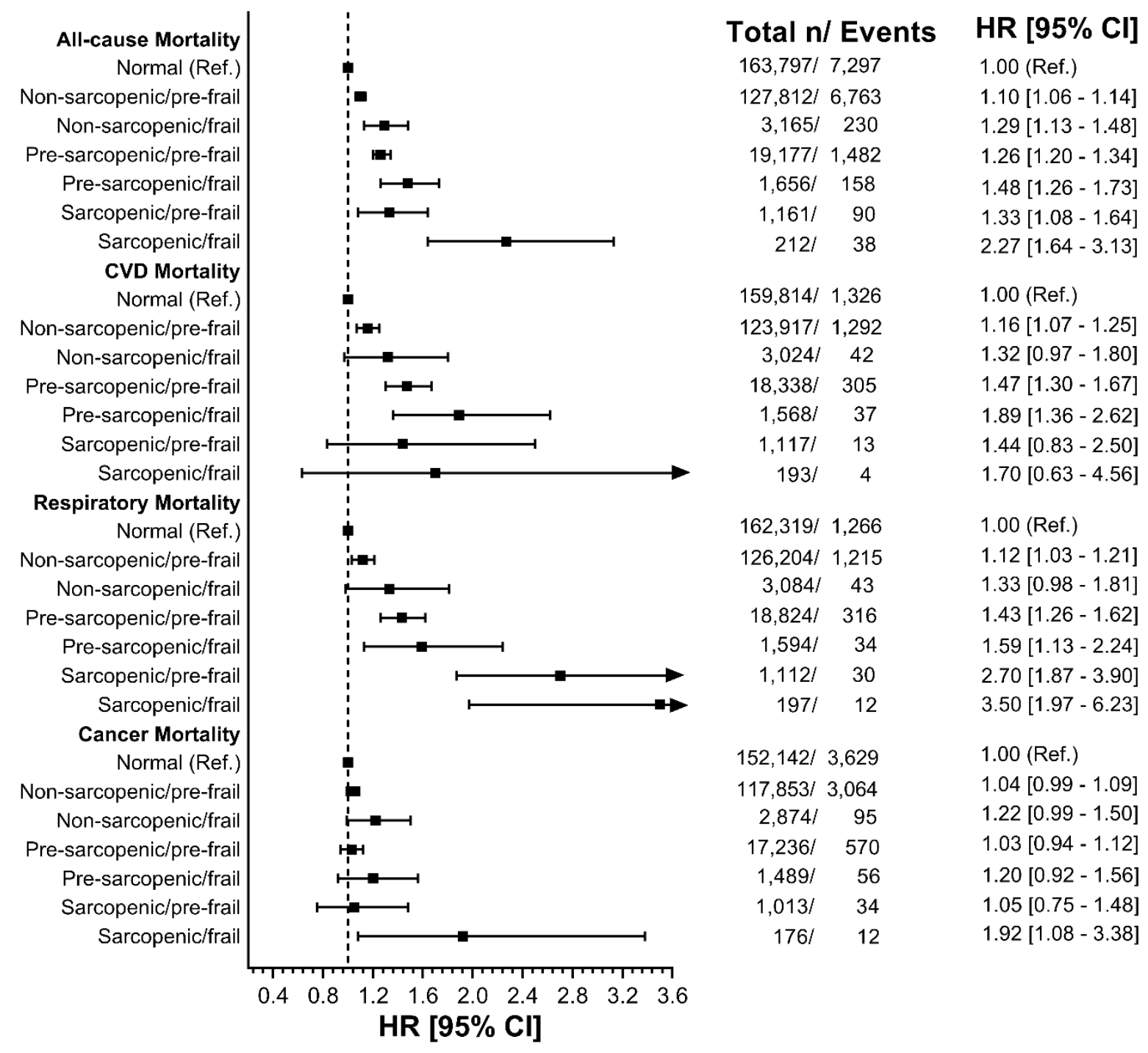

\section{Figure 3. The joint association of frailty and sarcopenia with all-cause and specific cause mortality}

Data presented as adjusted hazard ratio (HR) and its $95 \%$ confidence interval $(95 \% \mathrm{Cl})$ by combined categories of sarcopenia and frailty. People without sarcopenia or frailty were used as the reference group (label as normal in the graph).

All analyses were conducted excluding people with cancer $(n=24,197), \operatorname{CVD}(n=9,009)$ and respiratory disease $(n=3,646)$ at baseline when these were included in the outcome. Analyses were adjusted for age, sex, deprivation, smoking status, sleep duration, discretionary sedentary time, waist circumference, dietary intake (alcohol, red meat and processed meat intake), systolic blood pressure, diabetes, depression, comorbidities, as well as CVD, cancer, and respiratory disease, when these were not the outcome. Sarcopenic people include those with sarcopenia or severe sarcopenia. 